\title{
Pathological changes in the vagus nerve in diabetes and chronic alcoholism
}

\author{
Y-P GUO,* J G McLEOD, J BAVERSTOCK \\ From the Department of Medicine, University of Sydney, Australia
}

SUMMARY The pathological changes in the vagus nerve removed at necropsy have been studied in patients with chronic alcoholism and diabetes. Morphometric studies on myelinated fibres were performed on the nerve at mid-cervical, lung hilum and diaphragmatic levels. In two insulindependent diabetics the density of myelinated fibres was below the lower limit of the control range at all levels. In all the diabetic and chronic alcoholic subjects there was significant reduction in the density of myelinated fibres at the most distal level in the nerve. The degenerative changes in the nerve are consistent with the findings of abnormal vagal function in diabetics and chronic alcoholics.

Autonomic neuropathy is a well recognised complication of diabetes and to a lesser extent of chronic alcoholism. In diabetes there is frequently evidence of abnormal parasympathetic and sympathetic function on clinical testing, and abnormalities of the vagus usually become evident before those of the sympathetic system. ${ }^{1}$ Pathological changes have been described in both sympathetic and vagus nerves. ${ }^{2-8}$

In chronic alcoholism, postural hypotension and other manifestations of sympathetic dysfunction, apart from impaired sweating, are less common than in diabetes ${ }^{9}$ but may be present in Wernicke's encephalopathy ${ }^{1011}$ and in severe cases of alcoholic neuropathy. ${ }^{12}$ However, evidence of vagal damage may be manifested by impaired heart rate responses on autonomic testing before there is evidence of postural hypotension. ${ }^{13}$ Pathological changes have been demonstrated in sympathetic ganglia ${ }^{2}$ but the relatively normal density and fibre diameter distribution of myelinated fibres in splanchnic nerves contrast with the marked degenerative changes seen in diabetics. $^{69}$ In severe cases, degeneration in both vagus and sympathetic trunks has been described. ${ }^{12}$

There have been few pathological studies of the

* Present address: Department of Neurology, Peking Union Medical College Hospital, Chinese Academy of Medical Science, Beijing, Peoples' Republic of China.

Address for reprint requests: Professor J G McLeod, Department of Medicine, University of Sydney, NSW 2006, Australia.

Received 30 December 1986 and in revised form 18 March 1987. Accepted 26 March 1987 vagus nerve in patients with diabetic neuropathy ${ }^{3-578}$ and we are aware of only one in severe chronic alcoholism. ${ }^{12}$ The purpose of the present study was a systematic morphometric examination of the myelinated fibres in vagus nerve at different levels obtained at necropsy in patients with chronic alcoholism and in patients with diabetes.

\section{Methods}

The vagus nerve was removed at necropsy from 12 subjects who had no neurological illness, hypertension, diabetes or a history of alcoholism, from nine patients with chronic alcoholism and from four patients with diabetes. Post-mortem autolysis rendered many of the specimens unsuitable for detailed morphometric analysis and in the event the vagus nerve of four control subjects, three patients with diabetes mellitus (cases 1-3) and six chronic alcoholics (cases 4-9) were studied.

\section{Histological studies}

Necropsies were performed within 24 hours of death. Portions of the vagus nerve were removed at the mid-cervical level (level 1), the level of the lung hilum (level 2) and $3 \mathrm{~cm}$ above the diaphragm (level 3). From each portion one piece of nerve was prepared for semi-thin sections by fixing in $3 \%$ glutaraldehyde in $0.1 \mathrm{M}$ cacodylate butter for 3 hours followed by $2 \%$ osmium tetroxide for one hour. The specimens were dehydrated and graded in concentrations of ethanol and embedded in epoxy resin (Spurr's solution). Semi-thin sections $0 \cdot 5-1 \mu \mathrm{m}$ in thickness were stained with toluidine blue. A second piece of nerve was fixed in $10 \%$ formaldehyde, stained in $1 \%$ osmic acid for 24 hours and single nerve fibres teased out.

For morphometric studies of myelinated fibres photo- 
(a)
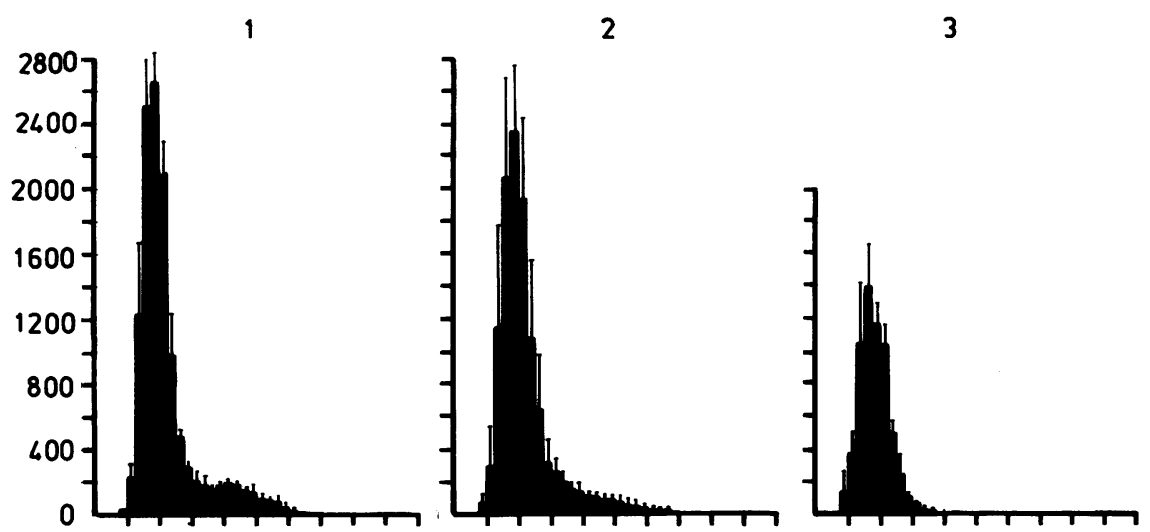

(b)

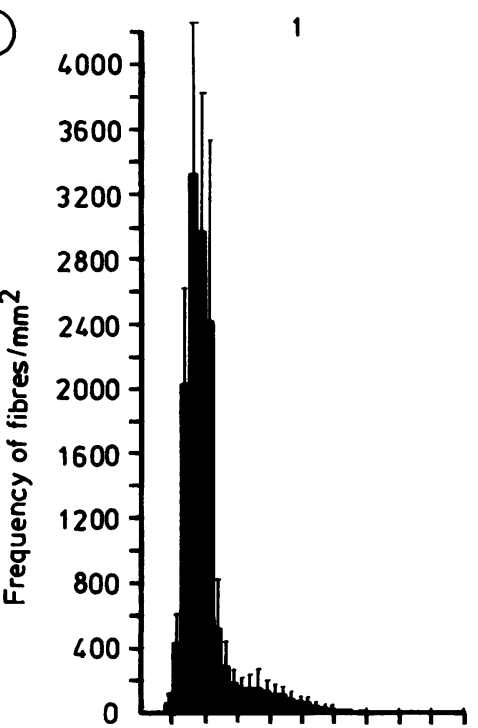

2
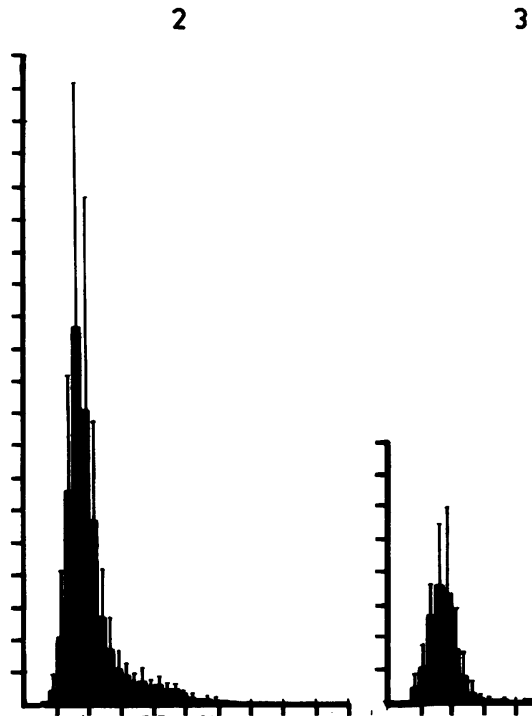

(c)

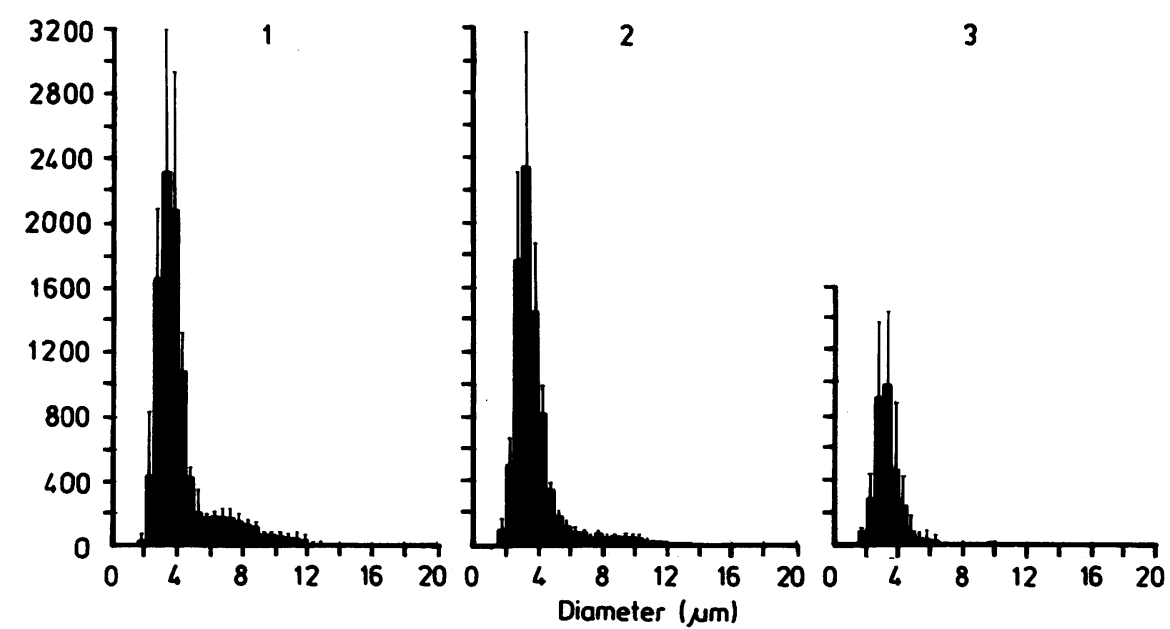

Fig 1 Mean myelinated fibre density distribution of vagus nerves from controls $(a)$, diabetics $(b)$ and alcoholics at level 1 (mid-cervical), level 2 (lung hilum) and level 3 (diaphragm). Bars indicate one standard deviation (c) from mean.

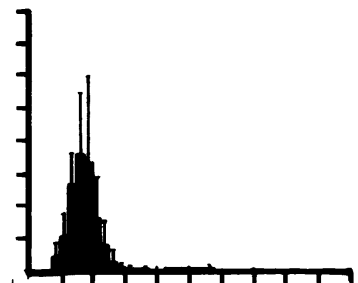

(1)

$\therefore$ 


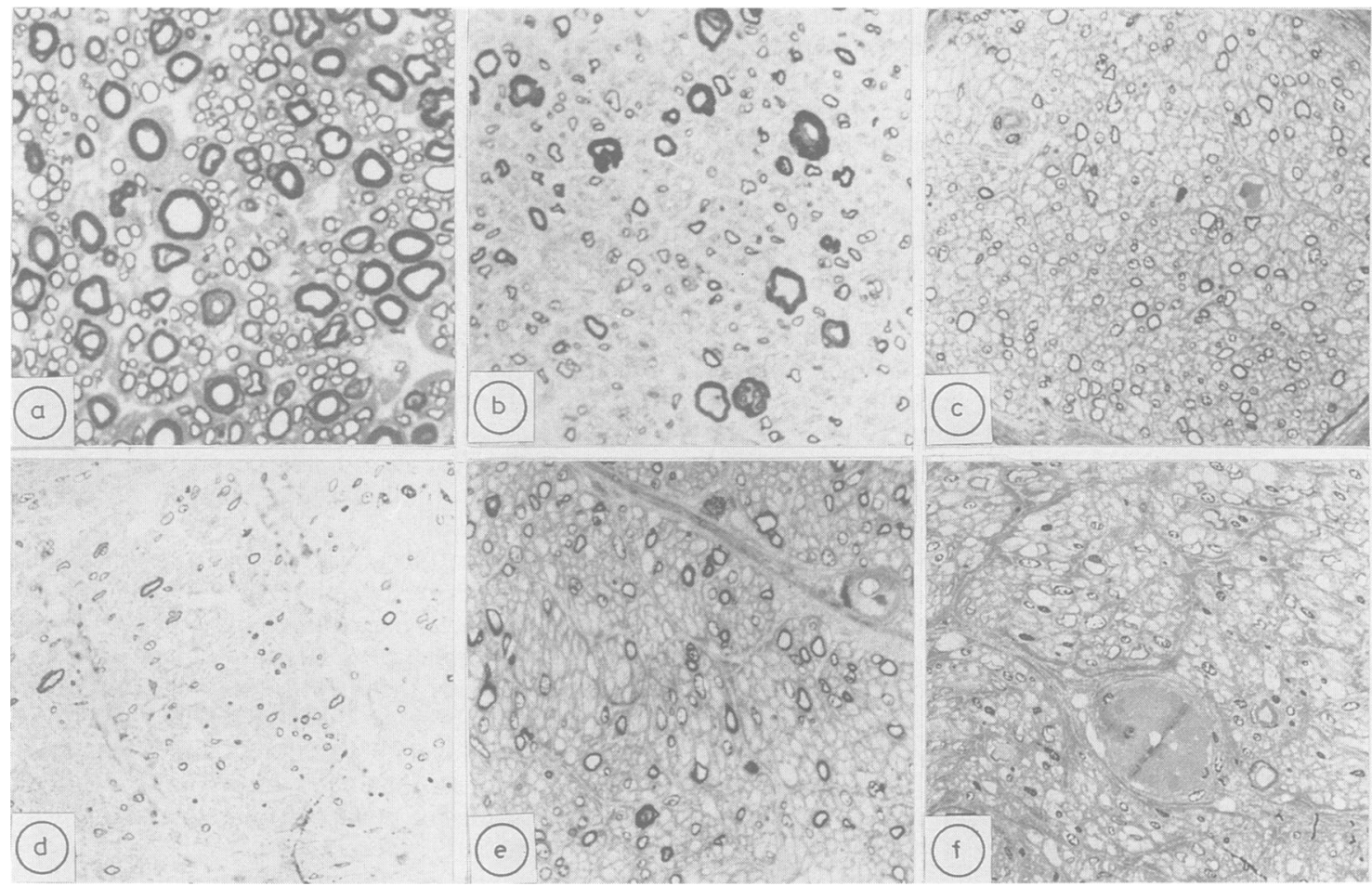

Fig 2 Transverse sections of vagus nerves of control (a) and diabetic case 1 (b) at level 2; control (c) and diabetic case 1 (d) at level 3; and control (e) and alcoholic case $9(f)$ at level 3. Bar $=50 \mu \mathrm{m}$. Toluidine blue.

micrographs of toluidine blue sections were made and selected fascicles printed on photographic paper at an enlargement of $\times 1100$. The diameter of myelinated fibres was measured using a Zeiss TGZ3 particle size analyser and histograms of the myelinated fibre distribution were constructed using a Hewlett Packard 9815A programmable calculator, digitiser and plotter and a program kindly made available by Dr PJ Dyck. The fibre densities were calculated as the number of fibres $/ \mathrm{mm}^{2}$ of intraperineurial area.

\section{Results}

\section{Clinical features}

Control subjects The ages of the four male control subjects ranged from 32 to 74 years (mean 57.5, SD 18.0). There was no history of diabetes, alcoholism or neurological disease. They died respectively of gastrointestinal haemorrhage from a peptic ulcer, pneumonia complicating chronic obstructive airways disease, drowning and head injury from a motor vehicle accident.

Diabetic subjects The ages of the three patients with diabetes (two males, one female) ranged from 56 to 81 years (mean 69.0, SD 12.5). Case 1 (male, 70) was an insulin-dependent diabetic for 4 years with a mild predominantly sensory peripheral neuropathy who died of a myocardial infarction. Case 2 (male, 76 ) had been a non-insulin-dependent diabetic for 12 years with a mild peripheral neuropathy who died of cerebral infarction. Case 3 (female, 81) had been an insulin-

Table Density of myelinated fibres (number of fibres $\left(\mathrm{mm}^{2}\right)$ in vagus nerve

\begin{tabular}{llll}
\hline & Level 1 & Level 2 & Level 3 \\
\hline $\begin{array}{l}\text { Controls } \\
\text { Mean }\end{array}$ & 12,322 & 11,532 & \\
SD & 2,543 & 1,792 & 6,201 \\
Range & $10,524-14,120$ & $10,274-14,131$ & 9,152 \\
n & 2 & 4 & 3 \\
& & & \\
Diabetic subjects & & & \\
Mean & 10,399 & 9,016 & 3,235 \\
SD & 1,213 & 3,742 & 1,119 \\
Range & $9,541-11,257$ & $5,059-12,498$ & $1,954-4,023$ \\
n & 2 & 3 & 3 \\
Significance & $\mathrm{p}>0 \cdot 25$ & $\mathrm{p}>0 \cdot 10$ & $\mathrm{p}<0 \cdot 01$ \\
Alcoholic subjects & & & \\
Mean & 12,678 & 9,062 & 2,878 \\
SD & 3,082 & 3,095 & 1,207 \\
Range & $11,024-17,299$ & $3,897-12,516$ & $2,735-4,140$ \\
n & 4 & 6 & 3 \\
Significance & $\mathrm{p}>0 \cdot 40$ & $\mathrm{p}>0.05$ & $\mathrm{p}<0.01$ \\
\hline
\end{tabular}


dependent diabetic for 9 years with a distal sensory neuropathy who died of ischaemic heart disease and bronchopneumonia.

Alcoholic subjects The ages of the six chronic alcoholic patients (four males, two females) ranged from 34 to 75 years (mean 61.7, SD 15.0). Case 4 (female, 60) was a longstanding chronic alcoholic with a past history of Wernicke's encephalopathy who died with carcinoma of the cervix and widespread metastases. Case 5 (male, 34) had a moderately severe alcoholic sensorimotor peripheral neuropathy, cirrhosis of the liver and died of hepatic failure. Case 6 (male, 74) was a chronic alcoholic with mild sensorimotor neuropathy who died in acute renal failure. Case 7 (male, 63) was a chronic alcoholic with a past history of Wernicke's encephalopathy who died with gastrointestinal haemorrhage complicating cirrhosis of the liver and portal hypertension. Case 8 (female, 75 ) died with hepatic failure and congestive cardiac failure. There was no clinical evidence of peripheral neuropathy. Case 9 (male, 63) had a mild peripheral neuropathy and died of myocardial infarction.

\section{Histopathological studies on the vagus nerve}

Controls At the mid-cervical level the vagus nerve consisted of a variable number of fascicles. Bundles of fibres destined for the recurrent pharyngeal nerve were superficially placed and contained a greater proportion of large diameter fibres than other fascicles.

The density of myelinated fibres at different levels is shown in the table and the myelinated fibre distribution in fig 1 . It may be seen that there is no significant difference in the densities of fibre diameter distributions at levels 1 and 2. At both levels there was a bi-modal distribution of fibres. The majority of fibres (greater than $80 \%$ ) were $6 \mu \mathrm{m}$ or less in diameter with a peak at $4 \mu \mathrm{m}$ and only a small proportion of fibres (less than $8 \%$ ) were $10 \mu \mathrm{m}$ or greater. At level 3 there was a unimodal distribution and most fibres (greater than $90 \%$ ) were $6 \mu \mathrm{m}$ or less with less than $1 \%$ of fibres being $10 \mu \mathrm{m}$ or more.

Diabetic subjects In cases 1 and 3 , both insulindependent diabetics, the density of myelinated fibres at all levels was below the lower limit of the control range. The histological appearance of the nerve from case 1 at two levels is shown in fig 2 . In all cases the density at level 3 was less than the lower limit of the control range and at this level the density of myelinated fibres for the whole group was significantly less than that of the control subjects $(p<0.01)$ (table). The predominant finding in teased fibre preparations at level 3 was axonal degeneration. Alcoholic subjects In all cases the density of myelinated fibres was in the control range at level 1 . In all cases the density of myelinated fibres at level 3 was below the lower limit of the control range and the mean density at this level was significantly less than that of controls $(p<0.01)$ (table). The histological appearance of the nerve of case 9 at level 3 is shown in fig 2 . The predominant finding in teased fibres at level 3 was axonal degeneration, more pronounced than at more proximal levels.

\section{Discussion}

With some exceptions, ${ }^{7}$ it has usually been necessary for histopathological examination of the human autonomic nervous system to be conducted on necropsy material. In the present study since suitable subjects were difficult to obtain and many of the nerves were unsuitable for morphometric analysis because of post-mortem autolysis, the number of nerves examined was small.

The histological structure of the vagus nerve in man has been previously described. ${ }^{14-17}$ About $80 \%$ of fibres at the mid-cervical level and at least $97 \%$ of fibres in the oesophageal plexus are unmyelinated. ${ }^{16}$ In the present study there was a bimodal distribution of myelinated fibres at the mid-cervical and lung hilum levels with over $80 \%$ of fibres being $6 \mu \mathrm{m}$ or less in diameter and less than $6 \%$ of fibres being $10 \mu \mathrm{m}$ or greater. Allowing for greater shrinkage in the preparations of the early workers with the different histological techniques, our findings are similar to those of Schnitzlein et al. ${ }^{15}$ In both studies few large diameter myelinated fibres were found at the diaphragmatic level since most of these large fibres are components of the recurrent laryngeal nerve. ${ }^{1517}$

The distribution of myelinated fibres in the humanc cervical vagus nerve is similar to that reported from our laboratory in the cat, ${ }^{18}$ guinea pig $^{19}$ and $\operatorname{dog}^{20}$ using similar techniques for fixation and morphometry, although there are differences in the density of fibres in the species.

The density of myelinated fibres in the vagus nerve in diabetic subjects at the diaphragmatic level was significantly reduced compared to controls and was reduced at all levels in the nerves of two patients. Teased fibre studies showed the changes were predominantly those of axonal degeneration. The reduced density of myelinated fibres is consistent with the observation of others, ${ }^{34}$ although these workers did not undertake quantitative studies and most of their patients had a longer history of diabetes and more severe peripheral neuropathy than the patients in our study. A severe reduction in density of unmyelinated fibres of the abdominal vagus nerve has been described in one insulin-dependent diabetic with gastroparesis. $^{7}$

In the subjects with alcoholic neuropathy there was a significant reduction in density of myelinated fibres in the distal part of the vagus when compared with 
controls. The more severe distal degeneration in the nerve and the pathological changes of axonal degeneration distally are consistent with the dying-back pathology described in this condition. ${ }^{21-23}$ Axonal degeneration of the vagus nerve was found at necropsy in four patients with severe alcoholic neuropathy who had hoarseness and weakness of voice and dysphagia. ${ }^{12}$ Morphometric studies have not previously been reported to our knowledge. In a previous study we were unable to demonstrate any significant morphological changes in the splanchnic nerve of alcoholic patients at necropsy. ${ }^{9}$ The degenerative changes in the vagus nerve are consistent with the findings of abnormal vagal function in alcoholics without postural hypotension. ${ }^{13}$

The work was supported by the Bushell Trust.

\section{References}

1 Watkins PJ, Edmonds ME. Clinical presentation of diabetic autonomic failure. In: Bannister R, ed. Autonomic Failure. Oxford: Oxford University Press 1983:337-70.

2 Appenzeller O, Richardson EP. The sympathetic chain in patients with diabetic and alcoholic polyneuropathy. Neurology 1966;16:1205-9.

3 Kristensson K, Nordborg C, Olsson Y, Sourander P. Changes in the vagus nerve in diabetes mellitus. Acta Pathol Microbiol Scand 1971;79A:684-5.

4 Duchen LW, Anjorin A, Watkins PJ, MacKay JD. Pathology of autonomic neuropathy in diabetes mellitus. Ann Intern Med 1980;92:301-3.

5 Duchen LW. Neuropathology of the autonomic nervous system in diabetes. In: Bannister R, ed. Autonomic Failure. Oxford: Oxford University Press 1983:437:52.

6 Low PA, Walsh JC, Huang C-Y, McLeod JG. The sympathetic nervous system in diabetic neuropathy. A clinical and pathological study. Brain 1975;98:341-56.
7 Guy RJC, Dawson JL, Garrett JR, Laws JW, Thomas PK, Sharma AK, Watkins PJ. Diabetic gastroparesis from autonomic neuropathy: surgical considerations and changes in vagus nerve morphology. J Neurol Neurosurg Psychiatry 1984;47:686-91.

8 Smith B. Neuropathology of the oesophagus in diabetes mellitus. $J$ Neurol Neurosurg Psychiatry 1974;37:1151-4.

9 Low PA, Walsh JC, Huang C-Y, McLeod JG. The sympathetic nervous system in alcoholic neuropathy. A clinical and pathological study. Brain 1975;98:357-64.

10 Gravellese MA, Victor M. Circulatory studies in Wernicke's encephalopathy. Circulation Res 19 1957;15:836-44.

11 Birchfield RI. Postural hypotension in Wernicke's disease. Am J Med 1964;36:404-14.

12 Novak DJ, Victor $M$. The vagus and sympathetic nerves in alcoholic polyneuropathy. Arch Neurol 1974;30:273-84.

13 Duncan G, Johnson RH, Lambie DG, Whiteside EA. Evidence of vagal neuropathy in chronic alcoholics. Lancet 1980;ii: 1053-6.

14 Hoffman HH, Kuntz A. Vagus nerve components. Anat Rec 1957;127:551-65.

15 Schnitzlein HN, Rowe LC, Hoffman HH. The myelinated component of the vagus nerves in man. Anat Rec 1958;131:649-67.

$16 \mathrm{Hoffman} \mathrm{HH}$, Schnitzlein HN. The numbers of nerve fibers in the vagus nerve of man. Anat Rec 1961:139:429-35.

17 Scheuer JL. Fibre size frequency distribution in normal human laryngeal nerves. J Anat (Lond) 1964;98:99-104.

18 Post EJ, McLeod JG. Acrylamide autonomic neuropathy in the cat. Part I. Neurophysiological and histological studies. J Neurol Sci 1977;33:353-74.

19 Tuck RR, Pollard JD, McLeod JG. Autonomic neuropathy in experimental allergic neuritis. An electrophysiological and histological study. Brain 1981;104:187-208.

20 Satchell PM, McLeod JG, Harper B, Goodman AH. Abnormalities in the vagus nerve in canine acrylamide neuropathy. J Neurol Neurosurg Psychiatry 1982;45:609-19.

21 Walsh JC, McLeod JG. Alcoholic neuropathy. An electrophysiological and pathological study. J Neurol Sci 1970;10: 457-69.

22 Behse F, Buchthal F. Alcoholic neuropathy: clinical, electrophysiological and biopsy findings. Ann Neurol 1977;2:95-110.

23 Victor M. Polyneuropathy due to nutritional deficiency and alcoholism. In: Dyck PJ, Thomas PK, Lambert EH, Bunge R, eds. Peripheral Neuropathy. Philadelphia: WB Saunders, 1984:1899-940. 